\title{
Piercing: Körpermodifikation oder Selbstverstümmelung
}

Arnd T. May

\section{Einleitung und Übersicht}

Menschen nehmen seit Jahrhunderten Veränderngen an ihrem Äußeren vor. Dabei werden vielfältige Modifikationen an den Haaren vorgenommen (Dauerwelle, Tönung, Entfernung von Locken, Einflechten von Echthaar, auszupfen, rasieren, schneiden), Wimpern verändert und teilweise werden künstliche Zähne getragen oder die natürlichen durch künstliche ergänzt, man

\begin{abstract}
„feilt diese an (Indonesien), schwärzt sie mit Ruß (Nordost-Indien), man trägt hohe Absätze, presst den Leib in Korsetts und feste Mieder, die Haut wird mit permanenten Make-up-Einlagen versehen, narbengezeichnet, tätowiert, durchstochen, Ohrlöcher werden erweitert, Schädel deformiert (Afrika), Hälse verlängert (Burma), Unterlippen erweitert (Afrika, Südamerika) und die Füße abgebunden (Japan, Burma, China)“.'
\end{abstract}

Körpermodifikationen können auf unterschiedliche Art und Weise erfolgen. Durch Schönheitsoperationen, zu denen zusätzlich zum obigen Katalog auch insbesondere Brustimplantate, Nasenkorrekturen, Fettabsaugen gehören, ${ }^{2}$ lassen Menschen ihren Körper verändern, um die Folgen des Alterns zu verbergen oder den Körper ihren ästhetischen Vorstellungen anzupassen. Einige dieser Körperveränderungen erfolgen aus therapeutischen Motiven, wenn z.B. durch Brustverkleinerungen die Belastung der Wirbelsäule verringert werden soll. In Einzelfällen erfolgen Körperveränderungen bei psychischem Leiden am eigenen Körper. Dieses durch Schönheitsoperationen gelinderte

1 Stirn (2002), S. 230.

2 Exemplarisch: Gilman (1999). 
seelische Leiden muss psychologisch-psychiatrisch diagnostiziert sein, damit die Kostenübernahme durch Krankenkassen erfolgt. So kann es im Einzelfall möglich sein, dass ein und dieselbe Operation von einer Patientin privat bezahlt werden muss und bei der Bettnachbarin von der Krankenkasse übernommen wird. Die Grenzen von Piercing und anderen Methoden der Körpermodifikation gegenüber der ,klassischen ästhetisch-kosmetischen Chirurgie (z. B. Brust-Silkonimplantate) “ verlaufen für Kaden/Bubenzer fließend. ${ }^{3}$ Ebenso ist die Abgrenzung von Körpermodifikationen zu pathologischen Phänomenen unscharf.

Neben Schönheitsoperationen werden Körperveränderungen vielfältig vorgenommen durch dauerhafte oder zeitweise vorgenommene Tätowierungen, Veränderungen der Hautoberfläche durch Brandmale oder Brandmarken (Branding) oder durch das Implantieren von körperfremden Materialien unter die Haut.

Nachfolgend werden Körpermodifikationen durch Piercings betrachtet. Dabei ist ein abgrenzendes Kriterium zu anderen Formen der Körpermodifikationen der nötige Einsatz von technischen Hilfsmitteln. Eine Tätowierung kann der Tätowierwillige zwar selbst mittels Nadeln vornehmen, aber wenn von professionellen Tätowierungen die Rede ist, so wird damit die Benutzung von Hilfsmitteln wie speziellem stromangetriebenen Werkzeug vorausgesetzt. Beim Piercing wird mit einem hautdurchbohrenden Hilfsmittel gestochen und in diese Durchbohrung wird ein Gegenstand eingesetzt. In Einzelfällen wie bei Sicherheitsnadeln verbleibt das penetrierende Werkzeug in der Haut. Die übliche Vorstellung von Piercings geht von einer Hautdurchbohrung mit folgendem Einsatz eines Provisoriums während der Heilungszeit aus oder dem Verbleib des eingesetzten Schmuckstücks.

Hautdurchbohrungen können im Gegensatz zu Tätowierungen praktisch überall durchgeführt werden, denn die nötigen Werkzeuge und Hilfsmittel für Piercings lassen sich einfach transportieren.

Ein möglicher Ort für das Piercen ist das ärztliche Behandlungszimmer in der Praxis oder einem Krankenhaus. Wenn Piercing für Zbinden die „Kunst [ist], sich kleine Löcher in den Körper zu stechen", ${ }^{4}$ so müssten auch Mediziner diese Fertigkeiten beherrschen. Aus der Vorstellung des Piercens durch Ärzte stellt sich die Frage, ob ihnen dies erlaubt ist. Eine Erlaubnis oder ein Verbot muss sorgfältig begründet werden. Dieser Frage geht der Beitrag nach, wenn einerseits das Selbstbestimmungsrecht des Kunden zu betrachten ist, der nach der bestmöglich ausgebildeten Person für das Piercen sucht, und einem sich möglicherweise durch das Piercen wandelnden Bild von Medizinern, die damit Körperverletzungen durchführen, für die es keine medizinische Indikation gibt.

Die Soziologin Degele sieht im Schönheitshandeln ein Medium der Kommunikation, welches „der Inszenierung der eigenen Außenwirkung zum Zweck der Erlangung von Aufmerksamkeit und Sicherung der eigenen Identität“ dient. Ferner ist für sie Schönheitshandeln „ein sozialer Prozess, in dem die

3 Kaden/Bubenzer (1998), S. 12.

4 Zbinden (1998), S. 9. 
Menschen versuchen, soziale (Anerkennungs-)Effekte zu erzielen, was freilich auch misslingen kann“. Je nach Betrachtungsweise kann die Körpermodifikation als positiv wahrgenommener Ausdruck der Individualität oder als Selbstverstümmelung bewertet werden. Schönheitsideale sind zeitspezifisch und kulturell beeinflussbar.

Die Bundesgesundheitsministerin hat sich 2005 u.a. mit der Beitragssammlung „Spieglein, Spieglein an der Wand. Zur Diskussion um den Schönheitswahn“" in die Diskussion eingebracht: „Man arbeitet an seinem Körper, aber man verletzt ihn nicht. " $M i t$ Verweis auf kosmetische Operationen stellt die Ministerin fest: „Kranke Menschen, die gerne gesund wären, müssen sich unfreiwillig Operationen unterziehen, während gesunde Menschen ihre Gesundheit riskieren. “8

Für die ethische Beurteilung des Piercens als ärztliche Tätigkeit werden gesellschaftliche Entwicklungen beschrieben, medizinische Komplikationen diskutiert und Vorschläge für Qualifikationen von Piercern dargestellt, um Hinweise für die mehrdimensionale Bewertung des Piercens zu geben. Dazu gehört auch die Frage nach der Reichweite und den Grenzen des Selbstbestimmungsrechts und der Forderung nach Eigenverantwortung in einer durch Fürsorgeleistungen geprägten Solidargemeinschaft.

\section{Entwicklungsgeschichte des Piercings und aktuelle Formen}

In Österreich wird Piercing durch die Ausübungsregeln für das Piercen und Tätowieren durch Kosmetik(Schönheitspflege)-Cewerbetreibende (Bundesgesetzblatt 14. Februar 2004, 647) auf Grund des $\mathbb{5} 69$ Abs. I der Gewerbeordnung 1994 (GewO 1994), BGBI. Nr. 194, zuletzt geändert durch das Bundesgesetz BGBI. I Nr. 111/2002, definiert als

„das Durchstechen der Haut zwecks Anbringung von Schmuck an Hautfalten, verknorpelten Stellen des Ohres oder des Nasenflügels, oder an der Zunge vor dem Zungenbändchen, sofern dazu ein Gerät verwendet wird, das höchstens zwei Millimeter durchmessend in die Haut eindringt und keine strich- oder flächenförmigen Verletzungen oder Vernarbungen verursacht".

Piercings können eine schmückende Funktion haben oder auch stimulierend wirken. Vor der Beschreibung von Piercings in der Gegenwart sind solche schon in früheren Zeiten vorgenommen worden.

Bereits römische Zenturionen haben ihre Brustwarzen durchstochen. Bei den Mayas wurden Zungen aus spirituellen Cründen gepierct. Im alten Ägypten galten Nasenringe als Statussymbol und waren der Oberschicht vorbehalten. Kulturell und spirituell motivierte Hautverzierungen (Bemalungen und dauerhafte Veränderungen) haben eine lange Tradition. Vereinzelt werden

5 Bundesministerium für Gesundheit und Soziale Sicherung (2005), S. 32.

6 Bundesministerium für Gesundheit und Soziale Sicherung (2005), S. 5 f.

7 Ebd.

8 Ebd. 
bewusst Narben und Brandmale (branding) angebracht. Im Rahmen dieses Beitrags sollen ausschließlich Piercings betrachtet werden.

In Stammesgesellschaften kommen Körperdekorationen seit Jahrhunderten vor. Dabei sind Körperveränderungen Teil von Initiationsriten oder Zugehörigkeitsmerkmale. Körperdekorationen kommt sowohl für den Geschmückten als auch für den Zuschauer Bedeutung zu. ${ }^{9}$ Piercings sind in gesellschaftlichen Randgruppen vermehrt aufgetaucht, so wie Tätowierungen im 18. Jahrhundert mit Gefangenen, Matrosen, Fremdenlegionären und Prostituierten in Verbindung gebracht wurden. Bei Matrosen wird die Gewohnheit berichtet, eine Äquatorüberquerung durch einen zusätzlichen Ohrring anzuzeigen. ${ }^{10}$ Bei reisenden Zimmerleuten sind häufig Ohrringe anzutreffen, welche traditionell mit einem Nagel gestochen werden. Nach dem Ehrenkodex der Zimmerleute wurde einem Gesellen bei unzünftigem Verhalten durch z. B. Diebstahl der Ohrring aus dem Ohrläppchen gerissen. Die Bezeichnung als „Schlitzohr“ rührt aus dieser Zeit. Die Ohrringe dienten teilweise der finanziellen Absicherung des Begräbnisses und wurden als Wertgegenstände behandelt. Kulturelle Amulette in Form von Ohrringen sind z. B. in der Schweiz bei den Appenzellern zu finden. ${ }^{11}$ Das Durchstechen der Ohrlöcher hat breite Akzeptanz und ca. $80 \%$ aller amerikanischen Frauen haben durchbohrte Ohren. Schildbach geht von zwei bis drei Millionen Menschen mit Piercings aus, wobei er Ohrlöcher ausschließt. ${ }^{12}$

Piercings sind manchmal äußere Zeichen einer emotionalen Bindung und besonderen dokumentierten Verbundenheit, wie sie auch durch Freundschaftsringe oder traditionelle Eheringe angezeigt wird, „die durch das Blut und das Schneiden des Fleisches geschlossen wird“. ${ }^{13}$

Strametz und Püschel sehen die Motive des Piercings in

„der Freude an der eigenen Körpermodifizierung und der eigenen Körperschmückung [...], um aus subjektiver Sicht die Attraktivität und das Selbstbewusstsein zu erhöhen, Aufmerksamkeit zu erlangen und vielleicht auch ein wenig ihre Umwelt zu provozieren““ ${ }^{14}$

Das Tragen von Ohrringen am rechten Ohr wurde im 20. Jahrhundert als Erkennungszeichen für Homosexuelle und somit als Codierung einer Lebensauffassung oder zumindest einer sexuellen Präferenz benutzt. Piercings waren für Feige lange dem „schwulen oder heterosexuellen Sadomasochismus und der Fetischszene vorbehalten, durchweg also einem experimentierfreudigen Völkchen“, und Piercings wurden mit „absonderlichem Sex“ assoziiert. ${ }^{15}$ Eine andere Zuschreibung nimmt Zbinden vor, wenn sie Piercings mit Anhängern einer bestimmten Musikrichtung in Verbindung bringt. ${ }^{16}$ Der Einfluss pro-

9 Stirn (2002), S. 224.

10 Zbinden (1998), S. 24.

11 Zbinden (1998), S. 51.

12 Schildbach (1998).

13 Zbinden (1998), S. 25.

14 Strametz/Püschel (2001), S. 23.

15 Feige (2002), S. 92.

16 Zbinden (1998), S. 24. 
minenter Personen wird durch das „Madonna-Piercing“ deutlich, was in der Oberlippe gestochen wird, dort wo die Sängerin Madonna ein Muttermal hat. ${ }^{17}$ Zbinden berichtet von der Eröffnung des ersten Fachgeschäfts für Piercing und Intimschmuck in Los Angeles im Jahr $1975 .{ }^{18}$

In Europa traten Piercings ab 1975 verstärkt in den „Londoner Slums“ auf. Hier wurden Sicherheitsnadeln benutzt mit dem Ziel „zu provozieren und zu schockieren“. ${ }^{19}$ Für Stirn hat die Punk-Bewegung Ende der 1970er bzw. Anfang der 1980er Jahre das Piercing entwickelt, welches heute Modetrend ist.

„Zunächst durchstach man sich mit einfachen Sicherheitsnadeln, dann - wohl aus Gründen allergischer Reaktionen - mit zunehmend verfeinerten, edleren Legierungen aus Silber oder Titanium, die bis heute kalt und glatt - ganz anders als sonst getragener Schmuck - wirken. Dieses selbstverletzende Schmücken bedeutete gleichzeitig auch eine Verstümmelung und damit gesellschaftliche Stigmatisierung. ${ }^{\text {“20 }}$

Unter dem Abschnitt „Punks - Das bunte Elend“ nimmt Lotz eine Beschreibung von Punks vor:

\begin{abstract}
„Man bevorzugt genietete Lederkluft oder zerrissene Jeans, drapiert mit Buttons, Hundeketten und aufreizenden Emblemen; der grellbunte Irokesenschnitt, aber auch andere aufwendig hergestellte Haarkonstruktionen sind Teile eines Kopfschmucks, der durch die Vielfalt seiner Dekorationsmöglichkeiten der Phantasie keine Grenzen setzt, weder beim Schminken noch beim Einsatz von Ketten, Ringen, Klammer und Nadeln in Ohr, Augenbrauen und Nase. Selbst Zunge, Lippen und Brustwarzen - von anderen Körperteilen ganz zu schweigen - bleiben nicht verschont. Der geschmückte Körper wird zur Demonstration einer Gegenkultur, die so schockierend wie ihre Musik - Ausdruck der Ohnmacht in einer Wettbewerbsgesellschaft, die Menschen nach ,Anforderungsprofilen' katalogisiert und jeden durch dieses Raster fallen lässt, der den Normen nicht entspricht. Als Reaktion setzte die Punk-Szene, die Ende der Siebziger Jahre im England des Thatcherismus entstand, ihre eigenen Normen. [...] In der Punkbewegung wird keine Existenzform erkennbar, sondern ein Zustand der Ausweglosigkeit - angereichert mit Zynismus und Hohn, Provokation und Aggression, um die Verzweiflung zu unterdrücken. Indem der Punk durch Piercing seine Haut schmückt, aber auch verstümmelt und sich so stigmatisiert, macht er körperlich jenen seelischen Schmerz sichtbar, der sich mit dem Wortsinn des Begriffs punk verbindet: Abfall, Mist. “21
\end{abstract}

Die Punk-Bewegung drückt für Zbinden „die Dekadenz unserer Großstädte, die Angst vor der Umweltverschmutzung und vor der Atomkraft aus. " Gleichzeitig werden alle „sozialen, ethischen, politischen und sexuellen Tabus“ gebrochen. ${ }^{22}$ Wenn die Körperveränderung konträr zu sozialen Normen erfolgt, kann dies als „Akt der Rebellion gegen kulturelle Schönheitsauffassungen“ angesehen werden. ${ }^{23}$ Piercings werden manchmal als „Protest gegen das ver-

\footnotetext{
17 Feige (2002), S. 113.

18 Zbinden (1998), S. 70.

19 Krause/Bremerich/Sztraka (2000), S. 21.

20 Stirn (2002), S. 233.

21 Lotz (1997), S. $234 \mathrm{f}$.

22 Zbinden (1998), S. 61.

23 Stirn (2002), S. 232.
} 
meintliche Kleinbürgertum“ oder als Gegenkultur betrachtet. ${ }^{24}$ Oft ist für Zbinden ein Piercing auch eine „Ausdrucksform der Revolte oder eine Ablehnung der herrschenden Werte “. ${ }^{25}$

Jean Paul Gaultier machte nach Ansicht von Feige Piercings salonfähig und es wurde aus „der schockierenden Wut“ nun „exaltierte Mode“, die gesellschaftsfähig wurde. ${ }^{26}$ Dies scheint die Symbole der Punk-Rebellion zu „entschärfen “ und bewusst für Mode einzusetzen. ${ }^{27} \mathrm{Im}$ Jahr 1993 schickt Gaultier seine Models mit Tätowierungen und Piercings auf den Laufsteg.

Seit dem vermehrten Auftreten von Piercings haben diese „eine zunehmende soziale und gesellschaftliche Akzeptanz“ erlangt..$^{28}$ „Wagten es anfangs nur wenige ,Mutige', sich ,piercen" zu lassen, so hat dieser Modetrend inzwischen allgemeine Akzeptanz und beträchtliche Ausweitung erfahren. ${ }^{\text {“29 Die }}$ Kinder der 199oer Jahre finden sich schön, sind stolz auf den eigenen Körper und möchten auffallen. Dies ist Zeichen einer besonderen Körperlichkeit. ${ }^{30}$ Jugendliche scheinen sich für Folz mehr am Modetrend der Erwachsenen zu orientieren, als sich zu der Bewegung der modernen Primitiven zugehörig zu fühlen. ${ }^{31}$

Neben religiös motivierten Piercings werden die meisten Piercings nach Strametz/Püschel aus „Freude an der eigenen Körpermodifikation und Körperschmückung“ angebracht, „um aus subjektiver Sicht die Attraktivität und das Selbstbewusstsein zu erhöhen, Aufmerksamkeit zu erlangen und vielleicht auch um ein wenig ihre Umwelt zu provozieren “, ${ }^{32}$ aber auch zur Identitätsund Gruppenbildung. Kaden/Bubenzer sehen einen „SM-Hintergrund“ der Modewelle. ${ }^{33}$ „Bodypiercing ist als Phänomen ein Grenzgänger zwischen Lust und Schmerz." ${ }^{34}$

Während Piercings als gewünschte Form des Körperschmucks unabhängig von Profession und Alter bei allen sozialen Schichten in zunehmendem Maße zu finden sind, wird diese Form der Modifikation und Dekoration des Körpers als „milde, benigne Form der Selbstverstümmelung“ " gesehen. ${ }^{35}$

Durch Piercings sind die Gepiercten lange Zeit mit ihrem Körper beschäftigt, wobei das Piercingstechen selbst einen kurzen Moment dauert, aber die folgende Pflege bis zur Ausheilung teilweise Monate in Anspruch nimmt. Die Abheilzeit nach dem Stechen eines Piercings wird je nach Körperregion mit wenigen Wochen bis zu einem halben Jahr angegeben.

Piercings werden manchmal von Vergewaltigungsopfern und Opfern sexuellen Missbrauchs als Weg bezeichnet, ein neues Verhältnis zu ihrem Körper zu

24 Cavelius/Wuillemet (1999), S. 100.

25 Zbinden (1998), S. 25.

26 Feige (2002), S. 94.

27 Zbinden (1998), S. 10.

28 Hörle/Kuba (2002), S. 200.

29 Krause/Bremerich/Sztraka (2000), S. 21.

30 Ziegler/Zoschke (1995), S. 33.

31 Folz et al. (2000), S. 380.

32 Strametz/Püschel (2001), S. 23.

33 Kaden (1998), S. 12.

34 Ziegler (1995), S. 64.

35 Strametz/Püschel (2001), S. 22; Folz et al. (2000), S. 378. 
erhalten. Dabei sind für Stirn insbesondere Intimpiercings eine Möglichkeit einer selbstgewollten Läsion an der traumatisierten Körperstelle. ${ }^{36}$

Türcke sieht Piercings als „Akte der Bemächtigung“, der Selbstbemächtigung nach dem Motto: „Sentio, ergo sum“.37 Nach Stirn weisen selbst angebrachte Piercings Parallelen zu Selbstmanipulierern auf, die solange ihren Körper einschneiden, bis Blut austritt. ${ }^{38}$

Piercings werden mitunter gesehen als Metapher für die Botschaften über Aids, Veränderung und Tod.$^{39}$ Dazu nimmt Zbinden eine Anleihe bei der ihrer Meinung nach derzeit materialistischen Welt, in welcher der Körper - die Religion ersetzend - geweiht ist. So lebt für sie im übertragenen Sinne die magische Absicht des Piercings fort und der Körper wird „misshandelt, erotisiert, er wird gequält und wahrgenommen. Es wird versucht, ihn zurückzuerobern in der Absicht, eine scheinbare Kontrolle über die Welt zu erlangen. " ${ }^{40}$ So scheint es nur schlüssig, wenn Modern Primitives mit ihrem Körper jenen Gegenstand verändern möchten, über den sie „noch Macht“ besitzen. ${ }^{41}$

Die verflüchtigte physisch ungreifbare mikroelektronische Welt produziert die Sehnsucht nach „, haptischer Erfahrung“ und den Wunsch nach Halt. Diesen Halt bieten Piercing, die nicht nur flüchtig reizen. Im Piercing hat die Sehnsucht für Türcke einen „Ausgleich“, ein „sozial gut verträgliches Ventil gefunden“, und die „prometheische Wut“ artikuliert sich verschämt. ${ }^{42}$

Es können an vielfältigen Körperstellen Piercings vorgenommen werden. Für Feige ist der Bauchnabelstecker der „Klassiker“ unter den Piercings. ${ }^{43}$ Dieser ist für Zbinden durch den Einfluss von Modeidealen insbesondere bei Jugendlichen und „Mode-Opfern“ beliebt. ${ }^{44}$

„Heute gehört es schon fast zum Standard, als Frau einen Nabelstecker, als Mann ein Brustwarzenpiercing zu tragen. Wer mehr auffallen will, lässt sich ein Augenbrauenpiercing machen. Wer sexuell neue Wege beschreiten möchte, hat ein Zungen- oder, noch schöner, Intimpiercing. Wer weiß heutzutage schon so genau, was der nette Schalterangestellte um die Ecke alles unter seinem Anzug trägt? “45

Vereinzelt wird die angebliche appetitzügelnde Wirkung eines Zungenpiercings und „Bereicherung [der] körperlichen Beziehung“ durch selbiges angegeben. ${ }^{46}$

Eine Befragung von 481 Studierenden 2001 in den USA zeigte eine Piercingquote bei Studenten von $42 \%$ und bei Studentinnen von $60 \%$. Bei den Studenten gaben $38 \%$ Ohrpiercings an, und $4 \%$ hatten sich die Zunge piercen

\footnotetext{
36 Stirn (2002), S. 230.

37 Türcke (2002), S. 73 (im Original kursiv).

38 Stirn (2002), S. 229.

39 Zbinden (1998), S. 10.

40 Zbinden (1998), S. 12.

41 Zbinden (1998), S. 75.

42 Türcke (2002), S. 74.

43 Feige (2002), S. 99.

44 Zbinden (1998), S. 89.

45 Feige (2002), S. 94.

46 Roth/Plötz (1998), S. 690.
} 
lassen, wobei sich davon die Hälfte das Piercing zum Zeitpunkt der Umfrage bereits entfernt hatte. $3 \%$ der Studenten hatten ein Brustwarzenpiercing, was jedoch drei Viertel der betreffenden Personen bereits entfernt hatten. Im Vergleich gaben $29 \%$ der befragten Studentinnen Ohrpiercings an, wobei diese Piercings nicht als Ohrlöcher im herkömmlichen Sinne für Ohrringe definiert waren. $16 \%$ hatten sich die Zunge piercen lassen und zur Hälfte bereits wieder entfernt, $6 \%$ hatten sich die Brustwarzen piercen lassen und $32 \%$ gaben Bauchnabelpiercings an, von denen knapp $10 \%$ wieder entfernt waren. ${ }^{47}$ In der gleichen Befragung wurde bei Studierenden der verschiedenen Semester eine Piercingquote zwischen 41 und 58\% erhoben, wobei zwei Studenten der Gesamtgruppe von 218 ein Intimpiercing angaben sowie vier Studentinnen von insgesamt 228 Befragten. ${ }^{48}$ Die erhobene Komplikationsrate der Gesamtgruppe gibt Mayers mit 9,2\% an. ${ }^{49}$ In einer Studie mit Teilnehmern eines Erziehungsprogramms für Jugendliche in den USA wurden bei $29 \%$ Tätowierungen erhoben und $69 \%$ gaben Piercings an. Die Zahl der Piercings pro Befragtem lag bei bis zu 18. Gemeinsam benutzte Nadeln $(1,5 \%)$ und andere unprofessionell vorgenommene Piercings werden von $20 \%$ der Befragten berichtet. ${ }^{50}$

Vermehrt wird von Wünschen nach Piercings im Zuge eines medizinischen Eingriffs mit Vollnarkose berichtet, da dann das Piercing garantiert schmerzfrei gestochen wird. In der Reaktion der Freundinnen und Freunde einer bei einer Operation gepiercten 22-jährigen Patientin wurde ihr für ihren Mut Bewunderung ausgesprochen. ${ }^{51}$

Nach der Präambel zur Leitlinie „Anforderungen der Hygiene beim Tätowieren und Piercen“ des Arbeitskreises „Krankenhaus- \& Praxishygiene“ der Arbeitsgemeinschaft der wissenschaftlichen medizinischen Fachgesellschaften (AWMF) ist unter Bezug auf die Rechtsabteilung der Bundesärztekammer Piercing keine ärztliche Tätigkeit. ${ }^{52}$ Dies ergebe sich aus dem obersten ärztlichen Gebot „primum nihil nocere“ sowie aus dem Gelöbnis des Weltärztebundes.

Der gewerblichen Tätigkeit von Ärzten neben ihrer Tätigkeit der Krankenversorgung in der Praxis oder Krankenhaus sind für Dierks Crenzen gesetzt, die in der Gefährdung des Ansehens der Ärzteschaft in der Öffentlichkeit zu sehen sind. Als Beispiel führt Dierks die Tätigkeit als Sargtischler oder Bordellbetreiber an. ${ }^{53}$ Die Durchführung von Piercings in Praxisräumen und nicht in einem separaten Raum könnte einen Verstoß gegen die werbliche Wechselwirkung zwischen Praxis und Gewerbe darstellen, wenn durch das Angebot von Piercings als komplementärer Leistung die Praxis für Patienten attraktiver wird.

Eine Behandlung durch den Arzt muss vom Patienten erlaubt werden, indem er nach dem Grundsatz der Einwilligung nach Aufklärung die Entscheidung zur Behandlung selbst trifft. Trotz Einwilligung des Patienten kann eine Behandlung strafbar sein, wenn diese gegen die „guten Sitten“ verstößt. Die-

47 Mayers et al. (2002), S. 31

48 Ebd.

49 Mayers et al. (2002), S. 33.

50 Braithwaite et al. (2001).

51 Ziegler/Zoschke (1995), S. 33.

52 Arbeitskreis „Krankenhaus- \& Praxishygiene“ der AWMF (2004), S. $148 \mathrm{ff}$.

53 Dierks (2003). 
ser unbestimmte Rechtsbegriff muss im Einzelfall konkretisiert werden. Das Reichsgericht hat bereits 1912 ausgeführt: „Ein Verstoß gegen die guten Sitten liegt nach ständiger Rechtsprechung vor, wenn eine Handlung dem Anstandsgefühl aller billig und gerecht Denkenden zuwiderläuft. " ${ }^{54}$ Was aber bedeutet dies konkret für das Stechen von Piercings durch Ärzte? Der Hinweis des Verbots einer freiwilligen Körperverletzung bei Verletzung der guten Sitten ist wenig konkret und hilfreich. ${ }^{55}$

Der Bundesgerichtshof stellt 2004 in einem Urteil fest, dass einverständlich vorgenommene sadomasochistische Praktiken, die zu Körperverletzungen führen, nicht als solche gegen die „guten Sitten“ im Sinne von $\$ 228$ StCB verstoßen. Als sittenwidrig im Sinne von $\mathbb{2} 228$ StGB bewertet der Bundesgerichtshof jene Taten, bei denen bei vorausschauender objektiver Betrachtung der Einwilligende durch die Körperverletzungshandlung in konkrete Todesgefahr gebracht wird. ${ }^{56}$ Der Senat des Bundesgerichtshofs sieht frühere anderslautende Entscheidungen des Reichsgerichts infolge gewandelter allgemeiner Moralvorstellungen als überholt an. Maßgebend für das Sittenwidrigkeitsurteil im Bezug auf sadomasochistische Handlungen sind Grad und Gewicht der drohenden Rechtsgutsverletzung. ${ }^{57}$

Ob bei Piercings die konkrete Todesgefahr bei vorausschauender objektiver Betrachtung ebenso anzunehmen ist wie bei der einvernehmlich stattgefundenen Strangulation bleibt fraglich. In einem graduellen Analogieschluss ist davon auszugehen, dass Piercings nicht gegen die guten Sitten im Sinne von $\$ 228$ StGB verstoßen.

In Deutschland bewertet z. B. die Ärztekammer Nordrhein Piercen als „nicht sinnvoll“ und „aus berufsethischer Sicht als unärztlich“. ${ }^{58}$ Der Justitiar der Ärztekammer Nordrhein geht aber nicht so weit, dass piercenden Ärzten die Approbation entzogen werde, denn das ist nur nach strafbaren oder „unwürdigen“ Handlungen möglich. Ein Eintrag im Telefonbuch „Piercing by the Doctor - Körperschmuck vom Fachmann“ wurde vom Ärztlichen Berufsgericht wegen Verstoß gegen das Werbeverbot mit einer Strafe belegt. ${ }^{59}$

Nach Feige ist es Ärzten nicht nur verboten, Piercings zu stechen, sondern auch dazu zu beraten. ${ }^{60}$ In der Wahrnehmung von Piercern sind die durch Ärzte gestochenen Piercings ,enttäuschend schlecht“ und nur aus finanziellen Motiven angeboten. ${ }^{61}$ Für Roth sind „,niedergelassene kosmetische Chirurgen ... eigentlich die besten Piercer“. ${ }^{2}$

\footnotetext{
54 Reichsgericht, RGZ, 1912, 80. Band, 221

$55 \bigcirc 228$ Strafgesetzbuch (Einwilligung): Wer eine Körperverletzung mit Einwilligung der verletzten Person vornimmt, handelt nur dann rechtswidrig, wenn die Tat trotz der Einwilligung gegen die guten Sitten verstößt.

56 Bundesgerichtshof, Urteil vom 26.05.2004 - 2 StR 505/03.

57 Bundesgerichtshof, Pressemeldung Nr. 60/2004 vom 26.05.2004 zur Strafbarkeit sadomasochistischer Praktiken mit tödlichem Ausgang.

58 Scheuer/Lehnen (2002).

59 Meyer (2001), S. 819.

60 Feige (2002), S. 129.

61 Ziegler/Zoschke (1995), S. 47.

62 nach Kaden/Bubenzer (1998), S. 13.
} 


\section{Behandlungsrisiken durch medizinische Komplikationen des Piercings}

Bei medizinischen Behandlungen kann es zu Komplikationen durch Piercings kommen, wenn diese den normalen Ablauf der Behandlung verzögern. Im idealen Fall kann der Patient die Piercings selbst entfernen oder Hinweise zu deren Entfernung geben. Dabei sind mehr als 90\% der Piercings durch Schraubbewegungen zu entfernen. Bei der Einleitung einer Narkose kann ein Zungenpiercing stören, und somit besitzt ein Zungenpiercing ein realistisches Gefahrenpotential. ${ }^{63}$ Die Aussagekraft radiologischer Untersuchungen kann durch belassene Piercings vermindert sein. Es können auch Brandverletzungen durch starke Erwärmungen auftreten, wenn Piercings nicht entfernt wurden. Die Entfernung von Piercings muss mit Patienten vor Beginn der Operation besprochen werden und falls die Entfernung zu schmerzhaft ist, muss das Einverständnis des Patienten eingeholt werden zur Entfernung unter Narkose. Bei Gefangenen sind Tätowierungen und Piercings als Risiken einer HepatitisInfektion beschrieben worden. ${ }^{64}$ Armstrong hat 1995 kaum medizinische Literatur und keine Artikel in Pflegezeitschriften der USA gefunden. ${ }^{65}$

Die prozentualen Zahlen zu Komplikationen bei Piercings werden unterschiedlich angegeben, und 1971 berichteten Cortese und Dickey von einer Komplikationsrate von $52 \%$ bei Ohrlöchern. ${ }^{66}$ Es kann zu einer Kelloidbildung als benigne, fibröse und proliferative Erkrankung kommen ${ }^{67}$ Für den Arbeitskreis „Krankenhaus- \& Praxishygiene“ der Arbeitsgemeinschaft der wissenschaftlichen medizinischen Fachgesellschaften (AWMF) wird das herkömmliche Ohrlochstechen nach der Präambel zur Leitlinie „Anforderungen der Hygiene beim Tätowieren und Piercen“ nicht unter Piercing subsumiert, da hierbei „im allgemeinen der Hygienestandard (sterile Nadeln, Hautdesinfektion) eingehalten wird“. ${ }^{68}$

Angesichts der hohen Komplikationsrate, einem möglicherweise entstehenden gesundheitlichen Schaden mit folgenden Kosten für das Versicherungssystem beim Piercen wird bezweifelt, dass diese Form des „nicht-medizinischen“"Eingriffs für Ärzte ethisch vertretbar ist. Jedoch kennen sich Mediziner mit den möglichen Komplikationen aus und verfügen über notwendige Kenntnisse der Hygiene, um möglichst Komplikationen zu vermeiden. Diese Kenntnisse liegen bei unseriösen Piercern „im Hinterzimmer“ nicht vor. Bei einer Befragung von 273 Patienten mit insgesamt 699 Piercings wurden Piercings ganz überwiegend von weiblichen Personen unter 25 Jahren gewünscht. Bei $28 \%$ der Befragten traten Komplikationen auf, die häufig zu Dauerschäden $(7,3 \%)$ bis hin zum mehrmonatigen Krankenhausaufenthalt nach Hepatitiserkrankung reichten. ${ }^{69}$ Als Gefahren des Piercings werden bakterielle und vira-

63 Roth/Plötz (1998), S. 691.

64 Braithwaite et al. (2001), S. 6.

65 Armstrong/Ekmark/Brooks (1995), S. 20.

66 Cortese/Dickey (1971), S. 66-72.

67 Mall/Pollmann/Müller (2002).

68 Arbeitskreis „Krankenhaus- \& Praxishygiene“ der AWMF (2004), S. $148 \mathrm{ff}$.

69 Krause/Bremerich/Sztraka (2000), S. 23. 
le Infekte bis zur Übertragung von Hepatitis B, C und D sowie HIV genannt. ${ }^{70}$ Teilweise traten bei Piercings der Zunge Atemwegsblockaden (Ludwigs-Angina) auf, die durch einen Luftröhrenschnitt (Tracheotomie) behandelt werden mussten. ${ }^{71}$ In Einzelfällen wird von einer Endokarditis nach Piercing berichtet. ${ }^{72}$ Nach Brustwarzenpiercing kann es zu einem vermehrten Milchfluss kommen. ${ }^{73}$ Neben der Irritation von Piercings durch Gleitmittel oder Spermicide sieht Muldoon ein erhöhtes Risiko für die Beschädigung von Kondomen durch Piercings. ${ }^{74}$ Peate verweist auf Gewebeschädigungen bei homosexuellen Sexualpartnern mit Piercing und rät zu besonderer Vorsicht. ${ }^{75}$ Die Beschreibung von Komplikationen nach Piercings bezieht sich auf Einzelfallbeschreibungen oder kleine Patientenkollektive, was die Angabe von aussagekräftigen Zahlen erschwert. Das Stechen von Piercings wird generell als eine sich „rasant ausbreitende, infektionsrelevante Innovation " angesehen. ${ }^{76}$

Peate betont die Notwendigkeit der Information, Schulung von Notfallpersonal in Kliniken im Umgang mit Piercings und der Entwicklung eines Verständnisses für modifizierte Anatomie. Beispielhaft wird das Einführen eines Katheters bei einem Patienten mit Genitalpiercing angeführt. ${ }^{77}$ Zur Entfernung von Piercings muss erforderliches Werkzeug vorhanden sein. Bethke und Reichart fordern, dass zahnärztliche Teams zur Beratung zu Piercings in der Lage sein sollten. ${ }^{78}$ Zahnärzte sollen auf mögliche Komplikationen vorbereitet sein. ${ }^{79}$ Ebenso wie Personal in Notaufnahmen müssen Praxisteams und Rettungsdienstpersonal entsprechend geschult und ausgerüstet sein.

\section{Vorgaben für nichtärztliche Piercer}

Verbindliche Vorgaben zur Qualifikation hat der Gesetzgeber nicht beschlossen und berufsrechtliche Vereinbarungen haben den Verbindlichkeitsgrad von Empfehlungen, die nicht sanktioniert werden können, wenn man vom Ausschluss aus einer unverbindlichen Gemeinschaft von Piercern absieht. Berufsrechtliche Regelungen sind unbekannt. Ein Piercer muss nach der Leitlinie „Anforderungen der Hygiene beim Tätowieren und Piercen“ des Arbeitskreises „Krankenhaus- \& Praxishygiene“ der Arbeitsgemeinschaft der wissenschaftlichen medizinischen Fachgesellschaften (AWMF) nach Punkt 2 über „ausreichendes medizinisches Wissen verfügen, um den Eingriff sachgerecht durchführen und auf Komplikationen adäquat reagieren zu können“". ${ }^{80}$ Diese abstrakte Formulierung bleibt in ihren Grenzen unscharf.

\footnotetext{
70 Bethke/Reichart (1999), S. 101.

71 Hörle/Kuba (2002), S. 201.

72 vgl. Strametz/Püschel (2001), S. 23; Handrick et al. (2003), S. 194.

73 Modest/Fangman (2002), S. $1626 \mathrm{f}$.

74 Muldoon (1997), S. 300.

75 Peate (2000), S. 2166.

76 Kistemann/Exner (2000), S. 253.

77 Peate (2000), S. 2163.

78 Bethke/Reichart (1999), S. 101

79 Sardella et al. (2002), S. 962.

80 Arbeitskreis „Krankenhaus- \& Praxishygiene“ der AWMF (2004), S. $148 \mathrm{ff}$.
} 
Das Verwaltungsgericht Gießen hat, bestätigt durch das Hessische Oberverwaltungsgericht Kassel, am 9. Februar 1999 (Az. 8 C 2161/98) entschieden, dass die inzwischen üblich gewordene Form des Piercens auch ohne die Verabreichung von Lokalanästhetika unter den Begriff der Heilkunde falle gemäß $\mathbb{1}$ Abs. 1 Heilpraktikergesetz (HPG) und somit nur von Personen mit ärztlicher oder heilkundlicher Fachausbildung durchgeführt werden darf, d. h. nur von Ärzten oder Heilpraktikern. Das Durchstechen unterschiedlicher Körperteile mit hochsensiblen Nervensträngen im Bereich der Zunge, den Augenbrauen und im Genitalbereich rechtfertig nach Ansicht des Verwaltungsgerichts die Auffassung des Piercings als Ausübung der Heilkunde, die nur mit einer ausdrücklichen Erlaubnis ausgeführt werden darf. Rudolph stellt nach der Entscheidung des Verwaltungsgerichts Gießen fest:

„Zweifelsfrei dürfte eine ,piercende Ärzteschaft' einen massiven Vertrauensverlust in der Bevölkerung erleiden und uns zu Recht von der 1. Stelle im Ansehen der Bevölkerung auf einen der unteren Ränge mitten zwischen die Politiker bringen. ${ }^{\star{ }^{81}}$

Er richtet eine dringende Mahnung an die Kostenträger, die Solidargemeinschaft nicht durch die Kostenübernahme beim Auftreten von Komplikationen nach Piercing zu belasten und dies auch in der Öffentlichkeit mit allem Nachdruck klarzustellen. ${ }^{82}$ Das Meinungsbild hierzu ist sicher noch nicht vollständig.

Piercer müssen bestimmte Auflagen beachten. Dazu gehören insbesondere Vorgaben zur Hygiene. Die Leitlinie „Anforderungen der Hygiene beim Tätowieren und Piercen“ wurde 2000 vom Arbeitskreis „Krankenhaus- \& Praxishygiene“ der Arbeitsgemeinschaft der wissenschaftlichen medizinischen Fachgesellschaften (AWMF) erstellt und im Februar 2004 überprüft. ${ }^{83}$ Unter dem dritten Abschnitt zu Beratung wird durch die Leitlinie „Anforderungen der Hygiene beim Tätowieren und Piercen“ gefordert, dass der „Kunde“ ausführlich und umfassend über alle Risiken und Folgen des jeweiligen Eingriffs zu informieren sei. ${ }^{84} \mathrm{Zu}$ den Räumen für die Durchführung von Piercings fordert die Leitlinie die gleichen Anforderungen, wie sie sich beim ambulanten Operieren ergeben. Die Dokumentationen zum Eingriff sind „in geeigneter Form und dauerhaft zu führen" und für 10 Jahre aufzubewahren" (Punkt 8. Nach dem Eingriff). ${ }^{85}$

Die Aufklärung des Patienten ist bei medizinischen Eingriffen und Untersuchungen obligatorisch vorgeschrieben, denn ohne Einwilligung ist der Eingriff rechtswidrig. Somit kann der Patient dem Arzt den Eingriff in seine körperliche Integrität, die Körperverletzung, genehmigen. Dazu muss der Patient die Einwilligung verstanden haben, was im Zweifel vom Arzt nachzuweisen ist. Zu den Erfordernissen der ärztlichen Aufklärungspflicht gehört die mit dem Eingriff intensiver und umfangreicher werdende Aufklärung je

81 Rudolph (2000).

82 Ebd.

83 Arbeitskreis „Krankenhaus- \& Praxishygiene“ der AWMF (2004), S. $148 \mathrm{ff}$.

84 Ebd.

85 Ebd. 
einschneidender und folgenreicher der Eingriff ist. Somit nimmt der Aufklärungsumfang mit abnehmender Dringlichkeit der Maßnahme zu, und dies gilt in besonderem Maße für „nur relativ oder gar nicht medizinisch indizierte Eingriffe, z. B. kosmetische Operationen“. ${ }^{86}$

Zur Einwilligungsfähigkeit stellte der Bundesgerichtshof 1969 fest, dass Einwilligende „die ausreichende Urteilsfähigkeit über Wesen, Bedeutung und Tragweite der gegen sie gerichteten Handlung " ${ }^{87}$ besitzen müssen. Einwilligungsfähigkeit wird vom BCH mit Einsichtsfähigkeit gleichgesetzt. Engisch beschreibt Einwilligungsfähigkeit als Besitz von „Reife und Fähigkeit [...], die Tragweite des ärztlichen Eingriffs für Körper, Beruf und Lebensglück zu ermessen“. ${ }^{88}$ Taupitz hat festgestellt, dass Einwilligungsfähigkeit nicht an Volljährigkeit gebunden ist und auch Minderjährige in der Regel ab Vollendung des 14. Lebensjahres Einwilligungsfähigkeit besitzen. ${ }^{89}$ Die individuellen Fähigkeiten sind im Einzelfall zu prüfen. Der Piercer muss sich überzeugen, dass der einwilligungsfähige Kunde die Aufklärung verstanden hat und geistig verarbeiten kann. In einer Entscheidung des Amtsgericht Neubrandenburg wurde einem Gepiercten ein Schmerzensgeld von knapp $300 €$ für aufgetretene Komplikationen nach unzureichender Aufklärung zugesprochen, wobei sich das verzögerte Aufsuchen eines Arztes vier Tage nach Auftreten der Komplikationen schmerzensgeldreduzierend auswirkte. ${ }^{90}$ Fragenkataloge im Vorfeld der Entscheidung zu einem Piercing werden von Muldoon ${ }^{91}$ und erweitert von Peate $^{92}$ vorgestellt. Dabei weist Muldoon auf die Notwendigkeit der ausreichenden und umfangreichen Information für Heranwachsende zu deren Unterstützung und Orientierung hin. ${ }^{93}$ Exemplarisch stellt Feige die Frage zur Selbstverantwortung von Piercinginteressierten, ob diese einem Gruppenzwang unterworfen sind oder das Piercing ,wirklich“ für sich selbst wollen. ${ }^{94}$

Der Petitionsausschuss des Deutschen Bundestages beschäftigte sich 2003 mit der Eingabe eines Vaters, der nach einem Bauchnabelpiercing seiner 13jährigen Tochter ohne seine Zustimmung nicht rechtlich gegen das Piercingstudio vorgehen könne. Dazu erklärte das Bundesministerium der Justiz, dass die Entscheidungskompetenz bei einem Piercing von der Einwilligungsfähigkeit abhängt, welche nicht an ein bestimmtes Alter, sondern an die geistige und sittliche Reife gebunden ist. ${ }^{95}$ Die Erste Organisation Professioneller Piercer e. V. (OPP) empfiehlt den 37 Mitgliedern, Piercings bei Jugendlichen erst ab 14 Jahren in Begleitung ihrer Eltern oder Erziehungsberechtigten zu stechen. Nach herrschender Meinung sind Kinder unter 14 Jahren nur in Ausnahmefällen einwilligungsfähig. ${ }^{96}$ Die vom Bundesgerichtshof entwickelte

\footnotetext{
86 Erlinger (2003), S. 628.

87 BGHSt 23, $1,4$.

88 Engisch (1958).

89 Vgl. Taupitz (2000).

90 Amtsgericht Neubrandenburg, Urteil vom 10.10.2000.

91 Muldoon (1997), S. 299.

92 Peate (2000), S. 2163.

93 Muldoon (1997), S. 299.

94 Feige (2002), S. 96.

95 dpa: „Können Eltern 13jährigen das Bauch-Piercing verbieten?, Ärztezeitung vom 3. Juli 2003.

96 Von Harder (2004), S. 1106.
} 
Stufentheorie gibt Aufschluss über die Beteiligung der Eltern im Vorfeld einer Behandlung bei Minderjährigen. ${ }^{97}$

Bei schönheitschirurgischen Eingriffen im Sinne von nicht zwingend indizierten Eingriffen ohne unmittelbaren Heilzweck muss der Patient vollständig und schonungslos aufgeklärt werden, ${ }^{98}$ damit er die günstigenfalls zu erwartenden Verbesserungen einem eventuellen Misserfolg, bleibender Entstellung und gesundheitlicher Beeinträchtigungen gegenüberstellen kann. Die zu fordernde Genauigkeit und Ausführlichkeit der Aufklärung steigt in dem Maße, in dem die therapeutische Erforderlichkeit abnimmt. ${ }^{99}$ Das Amtsgericht Neubrandenburg hat das Schmerzensgeld nach Komplikationen eines Zungenpiercings und unzureichender Aufklärung über die Risiken relativ niedrig angesetzt, da sich die Kundin „freiwillig einem Eingriff unterworfen hat, der allein der Mode und nicht der Heilung dient “. ${ }^{100}$ Der Verzicht auf die Aufklärung des Patienten in seinem Interesse als therapeutisches Privileg des Arztes sieht Giesen als überkommenes Privileg eines nicht mehr zu rechtfertigenden Paternalismus. ${ }^{101}$ Bezogen auf Piercings können ausführliche Informationen nur im Interesse des Kunden sein.

Entscheidungen werden im Gesundheitswesen oft unter Unsicherheit getroffen, die sich nach der Rational-Choice-Theory ${ }^{102}$ an der Nutzenfunktion und der Nutzenmaximierung des Entscheidungssubjekts orientieren. Die Bewertung einer Entscheidung als irrational beruht oft auf der Unkenntnis der entscheidungsleitenden Wünsche und Werte des Patienten.

Die Gesundheitsämter in Deutschland sollten den Kenntnisstand zu Hygienevorschriften und die Einhaltung selbiger überprüfen. Die Gewerbeanmeldung von Piercingstudios wurde an das Gesundheitsamt weitergegeben. Entsprechend durchgeführte Kontrollen von Tätowierern und Piercern seit 1995 in Frankfurt am Main ergaben zwischen 1995 bis 1997 eine sinkende Beanstandungszahl, doch nach dem Aussetzen der Kontrollen 1998 gab es mehr Beanstandungen. ${ }^{103}$

Dem Veranstalter einer „Convention“ kommt nach Feige eine hohe Verantwortung zu, denn Feige versichert in seinem Ratgeber, dass Veranstalter der Messen keine „halbseidenen Piercer“ zulassen würden und somit die „Seriosität" gesichert ist, da sonst die Veranstaltung in der Öffentlichkeit in Verruf geraten würde. ${ }^{104}$ Gleichwohl werden nach Feige auf einer Convention nur „schlichte Piercings“ gestochen. ${ }^{105}$ Die Ähnlichkeit von Piercingstudios zu Kliniken ist nach Feige ein Qualitätsmerkmal. ${ }^{106}$

97 Bundesgerichtshof (1988), S. 2946.

98 Bergmann, (2002), S. 4.

99 Vgl. Giesen (2000), S. 541.

100 Amtsgericht Neubrandenburg, Urteil vom 10.10.2000.

$101 \mathrm{Vgl}$. Giesen (2000), S. 541.

102 Das Individuum wählt jene Handlungsalternative, die ihm den größten Nutzen verspricht.

103 Heudorf/Kutzke/Seng (2002).

104 Feige (2002), S. 128.

105 Feige (2002), S. 129.

106 Feige (2002), S. 132. 
Für das Gesundheitsamt Bremen wurden Hygienestandards zur Infektionsprophylaxe in Tatoo- und Piercingsstudios entwickelt und mit einem Beratungsansatz zur Verbesserung der hygienischen Bedingungen ergänzt. ${ }^{107}$ Durch die Initiative des Gesundheitsamtes wurde die Informationsbroschüre des Gesundheitsamtes in mehr als drei Viertel der aufgesuchten Studios ausgelegt. ${ }^{108}$

Bei der Überwachung von Piercern sind bei der Kontrolle des Gesundheitsamtes das Lebensmittel- und Bedarfsgegenständegesetz (LMBG) und die entsprechende Ausführungs-Verordnung einschlägig, da insbesondere Nickel für Piercings verboten ist im Sinne des $\mathbb{3} 30$ LMBG. Teilweise kann es auch zu Korrosionserscheinungen durch verschiedene Metalle im Mund kommen. Piercingschmuck fällt zunächst nicht direkt in den Anwendungsbereich des Lebensmittel- und Bedarfsgegenständegesetzes (LMBG). Dessen $\mathbb{5} 5$ Abs. 3 ermächtigt aber das Bundesgesundheitsministerium, durch Rechtsverordnung andere Gegenstände des persönlichen Gebrauchs, von denen eine Gesundheitsgefahr ausgehen kann, den Lebensmitteln und Bedarfsgegenständen gleichzustellen. Mit Verordnung vom 10. April 1992 (BGBL I, 1992, S. $886 \mathrm{ff}$.) ist dies auch für „Ohrstecker oder gleichartige Erzeugnisse [...], die dazu bestimmt sind, bis zur Epithelisierung des Wundkanals im menschlichen Körper zu verbleiben“, klargestellt.

Für die Vornahme von Piercings muss geeignetes Material vorhanden sein. Eine 1999 in Frankfurt am Main durchgeführte Befragung von 57 Juwelieren ergab in 17 Fällen das Angebot von Ohrlöchern (11) und zusätzlich Nasenpiercings (6). Dabei wird häufig selbst bei Nasenpiercings eine Ohrlochpistole eingesetzt, was wegen der unzureichenden Desinfektion allein durch Einsprühen kritisiert wird. ${ }^{109}$ Durch Ohrlochpistolen wird die Haut zerstanzt und Gewebereste werden seitlich in den Stichkanal gedrückt, was die Infektionsrate erhöht. Ohrlochpistolen wurden ursprünglich nur zum Markieren von Rindern eingesetzt. ${ }^{110}$

Nach der Leitlinie „Anforderungen der Hygiene beim Tätowieren und Piercen“ des Arbeitskreises „Krankenhaus- \& Praxishygiene“ der Arbeitsgemeinschaft der wissenschaftlichen medizinischen Fachgesellschaften (AWMF) muss eine ,adäquate Nachsorge und Behandlung ggf. mit Überweisung zu einer entsprechenden Klinik oder Praxis bei Komplikationen [... ] jederzeit, auch nachts, sichergestellt sein". ${ }^{111}$ Die Erreichbarkeit am Abend oder am Wochenende sieht Feige bei Ärzten als nicht gegeben, wohl aber bei seriösen Piercern. ${ }^{112}$

Die Bekanntheit der bereits bestehenden Vorgaben für Piercings muss erhöht werden. Hierbei kommt Gesundheitsämtern und Gewerbeaufsichtsämtern eine tragende Rolle zu. Die berufsständische Selbstorganisation der Betreiber von Piercingstudios fällt derzeit gering aus. Die Leitlinie „Anforderungen der Hygiene beim Tätowieren und Piercen“ des Arbeitskreises „Kran-

107 Zolondek/Stelling/Hohmann (1998).

108 Zolondek/Stelling/Hohmann (1998), S. 172; Freie Hansestadt Bremen, Gesundheitsamt (2003).

109 Heudorf/Kutzke/Seng (2002), S. 223.

110 Folz et al. (2000), S. 378.

111 Arbeitskreis „Krankenhaus- \& Praxishygiene“ der AWMF, S. $148 \mathrm{ff}$.

112 Feige (2002), S. 129. 
kenhaus- \& Praxishygiene“ der Arbeitsgemeinschaft der wissenschaftlichen medizinischen Fachgesellschaften (AWMF) normiert Vorgaben, deren Einhaltung überprüft werden müssen. Die Ärztekammern sollten gemeinsam mit anderen Akteuren als Ansprechpartner für Piercinginteressierte, Betreiber von Piercingstudios und Piercer initiativ zur Verfügung stehen.

\section{Bewertung von Piercings im Spannungsfeld von Selbstverwirklichung und Fürsorge}

Piercings werden oft als Ausdruck der Selbstbestimmung und Selbstverwirklichung dargestellt. Dabei haben Modetrends in den letzten Jahren die Entscheidung der Kunden für ein Piercing beeinflusst. Mit Sorge wird dies bei Kindern und Jugendlichen betrachtet. Mit der Koalition gegen den Schönheitswahn hat die Bundesärztekammer im September 2004 eine Kampagne gegen die „Verführungen der Schönheits- und Werbeindustrie“ gestartet. Dies ist als Reaktion auf Schönheitsoperationen im Fernsehen vor einem Millionenpublikum zu sehen. Der Präsident der Bundesärztekammer, Jörg-Dietrich Hoppe, verurteilt suggerierte Defizite gegenüber Stars und Sternchen, über welche Kinder ihr Selbstwertgefühl definieren und damit die Schönheitschirurgie zum Jugendkult hochstilisieren. ${ }^{113}$ Weiter äußerte Hoppe erhebliche Zweifel an der Konformität des Handelns der betreffenden Ärzte mit dem Berufsrecht.

In seiner Rede zum 108. Deutschen Ärztetag in Berlin am 3. Mai 2005 betont der Präsident der Bundesärztekammer und des Deutschen Ärztetages, Professor Dr. Jörg-Dietrich Hoppe, die Verantwortung der behandelnden Ärzte, denn Patienten offenbaren sich in ihrer Not und ihrem Leid:

„Ethisch fragwürdig kann es allerdings werden, wenn nicht das medizinisch Sinnvolle, sondern - wie bei den so genannten Schönheitsoperationen - das ästhetisch Wünschenswerte in den Vordergrund rückt, wenn also die Ärzte als Body-Designer beansprucht werden. ${ }^{414}$

Hoppe äußert sich beunruhigt über den Anstieg der Zahl der ästhetisch-plastischen Operationen in den letzten Jahren. Insbesondere als Dokumentationen getarnte Reality-Shows zeigen für Hoppe, wie einfach Fettabsaugen, Falten glätten und Nasenkorrekturen sein sollen, und sie stellten diese Maßnahmen als erstrebenswerte Konsumgüter dar. Diese Schönheitsoperationsshows würden Scheinrealitäten konstruieren. Gerade Kinder und Jugendliche seien Opfer dieser Verführung zum Schönheitswahn.

Der Bundestag hat die gesetzlichen Crundlagen für ein Werbeverbot für Schönheitsoperationen geschaffen. Mit der 14. Novelle des Arzneimittelgesetzes (AMG) vom 29. August 2005 sind Schönheitsoperationen in den Anwendungsbereich des Heilmittelwerbegesetzes (HWG) einbezogen. Schönheitschirurgische Eingriffe, die nicht medizinisch notwendig sind, wie zum Beispiel Brustvergrößerungen durch Implantate oder Fettabsaugung zur Verbesserung der Körperformen, sind - wie jeder operative Eingriff - mit Risiken verbunden, 
die zu erheblichen Gesundheitsschäden führen können. Angesichts der rapide steigenden Zahlen von schönheitschirurgischen Eingriffen ist es daher für das Bundesgesundheitsministerium - wie im Fall von krankheitsbezogenen Eingriffen - notwendig, die Werbung für diese Verfahren dem Gesetz über die Werbung auf dem Gebiet des Heilwesens zu unterwerfen.

Der Vorsitzende der Deutschen Bischofskonferenz, Karl Kardinal Lehmann, sieht im neuen Trend zu Verschönerungen ein „Konsumgut“, was über rekonstruktive Hilfe nach Unfällen oder bei nachgewiesenen schwerwiegenden Leiden hinausgeht. ${ }^{115}$ Kardinal Lehmann begründet dies mit der dem Schönheitswahn zugrunde liegenden „Verzerrung des Menschenbildes“. Für die Katholische Kirche kommt jedoch die Menschenwürde jedem Menschen zu, auch unabhängig von dessen „äußerer Situation“. Der Mensch ist für die Kirche Ebenbild Gottes. Kardinal Lehmann sieht durch mögliche Abstufungen der Menschen eine Gefahr durch die „Verfügungsgewalt gesellschaftlicher Gruppen“ und sieht spezifischer bei jungen Menschen einen ,immensen Gruppendruck“, welcher sich in der für ihn irrigen Ansicht ausdrückt, dass die Machbarkeit für Jugendliche Ausdruck der Menschenwürde wird. Vermeintliche Selbstbestimmung wird für Kardinal Lehmann zur „Fremdbestimmung auf Grund von äußeren Einflüssen und gesellschaftlichem Druck “. ${ }^{116}$ Den gesellschaftlichen und individuellen Auftrag sieht Kardinal Lehmann mit Verweis auf den Theologen Guardini im Erkennen der „richtigen Balance zwischen Vernachlässigung seines Äußeren und einer übersteigerten Sorge allein um die leibliche Erscheinung “. ${ }^{117}$

Im Gesundheitswesen wird Menschen mit Piercings manchmal mit Ablehnung begegnet, wenn abweichende Wertvorstellungen und ablehnende moralische Intuitionen existieren. Badke beobachtet bei älteren Patienten eine Ablehnung von Piercings, da diese ähnlich wie Tätowierungen mit gesellschaftlichen Randgruppen assoziiert werden. Tätowierungen wurden oft durch „Zuchthäusler, Matrosen, Fremdenlegionäre, Gauner und leichte Mädchen“ im 18. Jahrhundert favorisiert. Punks als Randgruppe wurden nach Badke in den Medien häufig mit „kriminellen Gewalttaten, Drogenkonsum, sexueller Gewalt und Ausschweifungen in Verbindung gebracht“. ${ }^{118}$ In der Darstellung der Werbung dominiert hingegen der erotische und kosmetische Aspekt.

Berufsrechtlich empfiehlt sich eine räumliche Trennung der Tätigkeit als Arzt und als piercender Arzt. Piercings können nicht mit der Krankenkasse abgerechnet werden. Der Verkauf von Stickern, Piercing-Schmuck und sonstigem Zubehör in der Praxis ist berufsrechtlich für Ärzte nicht zulässig. ${ }^{119}$ Die Güte von vom Kunden mitgebrachten einzusetzenden Schmuck sollte überprüft werden. Im Zweifel sollte im Interesse der Sicherheit Schmuck mit festzustellender Güte und damit Unbedenklichkeit bevorzugt werden. Für Strametz/Püschel reichen mögliche Komplikationen selbst bei Schadensminimierung zur generellen Ablehnung von Piercings aus, da sie bezweifeln, dass ein Piercing

115 Kardinal Lehmann (2005), S. 12.

116 Kardinal Lehmann (2005), S. 13.

117 Kardinal Lehmann (2005), S. 14.

118 Badke (2001), S. 4.

119 Kaden/Bubenzer (1998), S. 14. 
Nutzen bringen kann. ${ }^{120}$ Das Vornehmen von Piercings durch Mediziner nach festgelegten Standards liegt im Interesse des nachfragenden Menschen. Für Roth sind „niedergelassene kosmetische Chirurgen [... ] eigentlich die besten Piercer“. ${ }^{121}$ Die Alternative zu Piercings durch Ärzte ist die Durchführung von Piercings von Menschen ohne klar festgelegte Kenntnisse. Manche Kunden möchten nach der Sensibilisierung für mögliche Risiken das Piercing vom „Fachmann“ vornehmen lassen, was aus unterschiedlichen Gründen nachvollziehbar ist. Nach Risikoabwägung bei Piercings ist der Wunsch nach einer kompetenten und gut ausgebildeten Person nahe liegend.

\section{Fazit}

Die gesellschaftliche Bewertung von Piercings ist von unterschiedlichen Einflussfaktoren und individuellen Ansichten abhängig. Piercings können im Gegensatz zu Tätowierungen ohne aufwändige technische Gerätschaften vorgenommen werden. Die zum Piercen notwendigen Hilfsmittel sind transportabel, frei verfügbar und nicht an einen bestimmten Ort gebunden. Der Ort der Vornahme des Piercings hat Einfluss auf das Infektionsrisiko.

In Bezug auf „Innovationen“ bei Piercings wird in Piercingmagazinen von „Conventions“ berichtet. Hierbei werden Oberflächenpiercings genannt, die als Haltepunkte für corsagenähnliche Bänder dienen. ${ }^{122}$ Mit den „üblichen“ Bauchnabelpiercings hat ein anderer Trend nichts gemein, bei dem sich Menschen an Haken durch die Haut am Rücken mit Seilen verbinden lassen. An diesen Seilen werden sie dann in die Höhe gezogen, bis sie frei schweben und das Gefühl haben, an sich selbst zu hängen. Diese besondere Körpererfahrung wird als „Superfly“ oder „Suspension“ bezeichnet. ${ }^{123}$ Für eine generelle Bewertung von Piercings sind die Arten der Hautdurchbohrungen hinsichtlich von Stichtechnik, Dauerhaftigkeit und die möglichen Begleitumstände des Stechens zu unterschiedlich.

Die Bandbreite von Piercings ist groß und fast unüberschaubar. Ebenso verschieden sind die Motive für Piercingmaßnahmen und die Reaktionen auf Piercings. Die ästhetischen Empfindungen sind bei Piercings unterschiedlich. Insbesondere ist bei Kindern und Jugendlichen der Wunsch nach Piercings präsent und dies erfordert zielgruppenorientierte Aufklärungskampagnen. Eine differenzierte Information trägt zum besseren Verständnis der Risiken bei. Information und Aufklärung wird als wesentliches Element der Infektionsverhütung angesehen. ${ }^{124}$ Die Wirkung sichtbarer Piercings sollte Gegenstand der Gesundheitserziehung in Schulen sein. Entsprechende Entscheidungsmodelle und -algorithmen aus den USA sollten auf ihre Anwendbarkeit in Deutschland überprüft und angepasst werden.

Eine Selbstorganisation von Piercern und Betreibern von Piercingstudios ist zum jetzigen Zeitpunkt nur ansatzweise festzustellen. Die Homepage der

120 Strametz/Püschel (2001), S. 24.

121 Nach Kaden/Bubenzer (1998), S. 13.

$122 \mathrm{Vgl}$. The art of Piercing, Ansbach (2005), S. $43 \mathrm{f}$.

123 Vgl. Huhn/Winkler (2005), S. 52-57.

124 Kistemann/Exner (2000), S. 253. 
Ersten Organisation Professioneller Piercer e. V. (OPP) enthält derzeit (Juni 2007) nur eine aussagelose Startseite ohne weitere Inhalte, die 2005 noch verfügbar waren.

Die European Association for Professional Piercing/Europäischer Berufsverband Professionelles Piercing e. V. verzeichnete Anfang 2007 knapp 100 Mitglieder und gibt die Zahl der Piercingstudios in Deutschland mit 7.000 an. ${ }^{125}$

Eine effektive Qualitätssicherung und Überprüfung von Hygienestandards ist fern der Realität. Angesichts von festgestellten Komplikationen auch bei in Studios gestochenen Piercings ist eine Kennzeichnung der Studios, bei denen bestimmte Standards garantiert werden, dringend geboten. Hierzu müssen rechtliche Vorgaben Berücksichtigung finden. Über die geltenden Bestimmungen hinaus sollten sich Betreiber von Piercingstudios und Berufsverbände der Qualitätsbeschreibung und Qualitätssicherung stellen. Denkbar ist ein transparentes Gütesiegel zur Ausbildung der Piercer und zur Einhaltung von Hygienestandards. Zur Information und Aufklärung sind einheitliche Standards hilfreich, was - in Analogie zur Medizin - die Einzelfallberatung und Aufklärung nicht ersetzen, wohl aber unterstützen kann. Nur durch differenziertes Informationsmaterial und eine umfangreiche Aufklärung ist die Basis für eine aufgeklärte Entscheidung eines einwilligungsfähigen Menschen herstellbar.

Die Ärztekammern sollten sich aktiv in die Erarbeitung von Kernkompetenzen für Piercer und ggf. in die Ausbildung von Piercern einbringen. Wenn Menschen einen hohen fachlichen Standard und Kompetenzen zur Vermeidung von Komplikationen wünschen, liegt der Gang zum Arzt nahe. In einer Befragung von u.a. medizinischem und pflegerischem Personal, Rettungsdienstpersonal und Schülern sprachen sich 68\% der 212 befragten Personen dafür aus, dass Ärzte Piercings stechen sollen. ${ }^{126}$ Für das Piercen durch Ärzte spricht die Abwendung von Schaden und Risiken durch professionelle Piercer, die mit ihrem medizinischen Wissen und unter optimalen hygienischen Bedingungen arbeiten können. Die Alternative zu Piercings durch Ärzte sind unter fragwürdigen Umständen gestochene Piercings. Ärzte sollten aus pragmatischen Überlegungen unter den oben skizzierten Rahmenbedingungen Piercings vornehmen dürfen, ohne dass ihnen standesrechtliche Sanktionen drohen. Ein Anspruch auf Piercings durch Ärzte lässt sich nicht ableiten. Eine Ablehnung des Stechens von Piercings steht jedem Arzt aus Gewissensgründen frei. Hier muss die individuelle moralische Ansicht des Arztes geschützt werden. Mittelfristig sollten entsprechende Rahmenbedingungen für Piercings und den Betrieb eines Piercingstudios transparent und effektiv überprüfbar sein. Diese Sicherheit dient dann den Kunden. Speziell Jugendliche haben ein besonderes Schutzbedürfnis.

Seit einiger Zeit wird über die Finanzierung von Lifestyle-Medizin und die Abgrenzung von Versicherungsleistungen der Solidargemeinschaft gegenüber nicht medizinisch indizierten Leistungen selbst diskutiert. Die Kosten für das Piercing selbst werden durch mögliche Komplikationen weit überschritten. 
Die Solidargemeinschaft muss sich verständigen, ob sie weiterhin die Kosten für Komplikationen bei selbst gestochenen Piercings übernehmen möchte. Durch das Gesetz zur Stärkung des Wettbewerbs in der gesetzlichen Krankenversicherung (GKV-Wettbewerbsstärkungsgesetz - GKV-WSG) vom 26. März 2007 wurde der $\$ 52$ im SGB V neu gefasst:

„(2) Haben sich Versicherte eine Krankheit durch eine medizinisch nicht indizierte Maßnahme wie zum Beispiel eine ästhetische Operation, eine Tätowierung oder ein Piercing zugezogen, hat die Krankenkasse die Versicherten in angemessener Höhe an den Kosten zu beteiligen und das Krankengeld für die Dauer dieser Behandlung ganz oder teilweise zu versagen oder zurückzufordern."

Die Bundesärztekammer fordert die politische Thematisierung des $\mathbb{5} 52 \mathrm{Abs} .2$ SGB V, da hiermit das „Verursacher-Prinzip“ in die GKV eingeführt werde. ${ }^{127}$ Konkrete Kataloge oder Hinweise zur Anwendung dieser normativen Vorgaben durch die Krankenkassen sind nicht bekannt. Somit bleibt für Gepiercte eine Unsicherheit, was die Höhe der „angemessenen“ Beteiligung an den Behandlungskosten bei Komplikationen betrifft.

Wenn nun die Solidargemeinschaft von der Kostenlast für Komplikationen entbunden werden soll, ist eine private Bezahlung durch den Gepiercten erforderlich. Denkbar wäre auch ein Modell der Zwangsversicherung beim Stechen eines Piercings, die Kosten im Fall möglicher Komplikationen abdeckt. Diese Versicherung soll fester Bestandteil der Dienstleistung und nicht optional buchbar sein. Diese Regelung verhindert eine Diskussion um die Sinnhaftigkeit der Versicherung: Hiermit wird der individuelle Gestaltungsspielraum des Kunden aus übergeordneten Gründen eingeschränkt. Das Argument der Verteuerung des Piercings und eine damit drohende Abwanderung zu Piercern, welche missbräuchlich die Zwangsversicherung nicht einschließen, muss im Rahmen der allgemeinen Abwägung betrachtet werden. Bereits heute nehmen Menschen aus unterschiedlichen Motiven Piercings selbst vor oder bitten andere Menschen ohne entsprechende Kenntnisse. Somit entziehen sie sich dem Versicherungsschutz und müssen die individuelle Kostenbeteiligung für Komplikationen akzeptieren. Die zusätzlichen Kosten nach dem Modell der integrierten Versicherung gegen Komplikationen für diese nicht abzuwählende Leistung richten sich für die Versicherungsunternehmen nach der Komplikationsquote und -schwere. Die Versicherer werden ein Interesse an der Reduktion von Komplikationen haben und vermutlich Impulse für eine Qualitätssicherung zur Vornahme von Piercings geben.

In der oben genannten Befragung von u.a. medizinischem und pflegerischem Personal, Rettungsdienstpersonal und Schülern befürworteten $73 \%$ die Eigenverantwortung des Gepiercten, der für mögliche Kosten für Komplikationen selbst aufkommen soll. Für 16\% der Befragten sollen Krankenkassen für Komplikationskosten aufkommen und für $48 \%$ der Befragten soll der „verursachende“

127 Entschließung des 110. Deutschen Ärztetags: 1. Erste Bewertung nach Einführung des GKV-Wettbewerbsstärkungsgesetzes (Drucksache I-01) (2007). 
Piercer die Komplikationskosten übernehmen. ${ }^{128} \mathrm{Zu}$ diesem Themenkomplex ist die Ausweitung der gesellschaftlichen Diskussion erforderlich.

Der Selbstbestimmung des Piercers und des Gepiercten kommt eine hohe Bedeutung zu. Um eine selbstbestimmte Entscheidung treffen zu können, müssen ausführliche Informationen verfügbar sein. Zur Erhöhung der Gesundheitsmündigkeit gehört auch die Möglichkeit der Auseinandersetzung mit Gesundheitsrisiken durch Piercings. Zusammen mit Informationsmöglichkeiten scheint eine altersunabhängige Zwangsversicherung ein mildes Mittel zur Vermeidung einer harten und möglicherweise kaum durchsetzbaren Altersgrenze zu sein. Für die Regulierung eines derzeit kaum regulierten und nicht effektiv kontrollierten Marktes spricht die Reduzierung von Komplikationen nach Piercings. Einen Anspruch auf ein Piercing haben Kunden nicht. Die Schutzpflichten des Staates und seiner Vollzugsorgane stehen dem Wunsch nach komplikationsarmen Piercings nicht entgegen, sondern ermöglichen diese derzeit erst. Subsidiäre Regelungen der Fürsorge sollten in regelmäßigen Abständen in Erwägung gezogen werden. Dabei muss dem Respekt vor der Selbstbestimmung der Kunden eine hohe Bedeutung beigemessen werden, die jedoch mit fürsorglich motivierten Vorgaben ausbalanciert werden muss.

Individuelle Bewertungen von Piercings sollten stets im gesellschaftlichen Kontext gesehen werden. Neben einem anhaltenden Trend zu Piercings bekennen einzelne Popstars wie Christina Aguilera medienwirksam das Ablegen der meisten Piercings, da für sie die Phase der Rebellion vorüber sei. ${ }^{129}$ Die Meinungsvielfalt zu Piercings ist ein konkretes Beispiel für unterschiedliche Ansichten und Bewertungssysteme, die Ausdruck der Selbstbestimmung des Menschen und Teil seiner Freiheit sind. Diese Freiheit muss respektiert werden. 


\section{Literatur}

Amtsgericht Neubrandenburg (2001): Amtsgericht Neubrandenburg, Urteil vom 10.10.2000, Neue Juristische Wochenschrift 2001, S. $902 \mathrm{f}$.

Arbeitskreis „Krankenhaus- \& Praxishygiene“ der AWMF (2004): Arbeitskreis „Krankenhaus- \& Praxishygiene“ der AWMF, Empfehlungen zur Hygiene in Klinik und Praxis, Anforderungen der Hygiene beim Tätowieren und Piercen, Hygiene in Klinik und Praxis, Wiesbaden 32004, S. $148 \mathrm{ff}$.

Armstrong/Ekmark/Brooks (1995): Myrna L. Armstrong, Elaine Ekmark, Barbara Brooks, Body Piercing: Promoting Informed Decision Making, Journal of School Nursing 11 (1995), p. 20-25

Barilan (2005): Michael Y. Barilan, The story of the body and the story of the person: Towards an ethics of representing human bodies and body-parts, Medicine, Health Care and Philosophy 8 (2005), p. 193-205

Bergmann (2002): Karl Otto Bergmann, Die Patientenaufklärung im Spiegel der Rechtsprechung des Jahres 2000, Arzt und Krankenhaus, 4 (2002), S. 3-6

Bethke/Reichart (1999): Gudrun Bethke, Peter A. Reichart, Risiken des oralen Piercings, Mund-, Kiefer-, Gesichtschirurgie (1999), S. 98-101

Braithwaite et al. (2001): Ronald Braithwaite, Alyssa Robillard, Tammy Woodring et al., Tattooing and body piercing among adolescent detainees: Relationship to alcohol and other drug use, Journal of Substance Abuse 13 (2001), p. 5-16

Bundesärztekammer (2004): Bundesärztekammer, Pressemeldung vom 25.09.2004, Gemeinsam gegen den Schönheitswahn

Bundesärztekammer (2007): Bundesärztekammer, Entschließung des110. Deutschen Ärztetags, Erste Bewertung nach Einführung des GKV-Wettbewerbsstärkungsgesetzes (Drucksache 1-01), www.bundesaerztekammer. de/downloads/DAETBeschlussprotokoll20070531.pdf

Bundesgerichtshof (1988): Bundesgerichtshof, Neue Juristische Wochenschrift (1988), S. 2946

Bundesgerichtshof (2004): Bundesgerichtshof, Urteil vom 26.05.2004 - 2 StR 505/03

Bundesgerichtshof (2004): Bundesgerichtshof, Pressemeldung Nr. 60/2004 vom 26.05.2004, Bundesgerichtshof zur Strafbarkeit sadomasochistischer Praktiken mit tödlichem Ausgang

Bundesministerium für Gesundheit und Soziale Sicherung (2005): Bundesministerium für Gesundheit und Soziale Sicherung (Hrsg.), Spieglein, Spieglein an der Wand. Zur Diskussion um den Schönheitswahn, Berlin 2005, S. 5 f.

Cavelius/Wuillemet (1999): Alexandra Cavelius, Sascha Wuillemet, Bodypiercing. Henna, Tattoos, Piercing \& Co., Augsburg 1999

Cortese/Dickey (1971): Thomas A. Cortese, R. A. Dickey, Complications of ear piercings, American Family Physician (1971), p. 66-72

Dierks (2003): Christian Dierks, Trennung zwischen Gewerbe und Praxis muss unmissverständlich sein, Ärztezeitung 09.04.2003

dpa (2003): dpa, Können Eltern 13jährigen das Bauch-Piercing verbieten?, Ärztezeitung 03.07.2003

dpa (2006): dpa, US-Popstar legt fast alle ihre Piercings ab, Westdeutsche Allgemeine Zeitung, 14.06.2006

Engisch (1958): Karl Engisch, Die rechtliche Bedeutung der ärztlichen Operation, in: Rudolf Stich, Karl Heinz Bauer (Hrsg.), Fehler und Gefahren bei chirurgischen Operationen, Jena, 4. Aufl., 1958, S. 1521-1557

Erlinger (2003): Rainer Erlinger, Die Aufklärung nicht Deutsch sprechender Patienten, Anästhesist 52 (2003), S. $625-629$

Feige (2002): Marcel Feige, Tattoo \& Piercing richtig gemacht. Ein Ratgeber für Einsteiger, Berlin 2002, S. 92129

Freie Hansestadt Bremen, Gesundheitsamt (2003): Freie Hansestadt Bremen, Gesundheitsamt, Tattoos und Piercing, sauber und sicher, Bremen 2003

Folz et al. (2000): Benedikt J. Folz, Burkard M. Lippert, Christoph Kuelkens et al., Hazards of Piercing and Facial Body Art: A Report of Three Patients and Literature Review, Annals of Plastic Surgery 45 (2000), p. 374381

Giesen (2000): Dieter Giesen, Einwilligung, in: Wilhelm Korff, Lutwin Beck, Paul Mikat (Hrsg.), Lexikon der Bioethik, Band I, Gütersloh 2000, S. 539-543 
Gilman (1999): Sander L. Gilman, Making the body beautiful. A cultural history of aesthetic surgery, New Jersey 1999

Handrick et al. (2003): Werner Handrick, Pietro Nenoff, Heidrun Müller et al., Infektionen durch Piercing und Tattoos - eine Übersicht, Wiener Medizinische Wochenschrift 153 (2003), S. 194-197

Von Harder (2004): Yvonne von Harder, Rechtliche Besonderheiten bei der Behandlung minderjähriger Patienten, Anästhesist 53 (2004), S. 1105-1110

Heudorf/Kutzke/Seng (2002): Ursel Heudorf, Gudrun Kutzke, Ursula Seng, Tätowieren und Piercing - Erfahrungen aus der infektionshygienischen Überwachung eines Gesundheitsamtes, Gesundheitswesen 62 (2002), S. 219-224

Hoppe (2005): Jörg-Dietrich Hoppe, www.bundesaerztekammer.de/25/15Reden/85Eroeffnung-108_DAET.html (2005)

Hörle/Kuba (2002): Steffen Hörle, G. B. Kuba, Komplikationen nach Brauenpiercing, Ophtalmologe (2002) S. 200-202

Huhn/Winkler (2005): Dieter Huhn, André Winkler, Suspension, Piercing 30 (2005), S. 52-57

Jacobs et al. (2002): Volker R. Jacobs, Kirstin Golombeck, Walter Jonat et al., Drei Fallbeispiele von Brustabszess nach Brustwarzenpiercing: Unterschätzte Gesundheitsprobleme eines Modephänomens, Zentralblatt für Gynäkologie 124 (2002), 378-385

Kaden/Bubenzer (1998): Marion Kaden, Rainer H. Bubenzer, Piercing: Dienstleistung vom Arzt?, Münchner Medizinische Wochenschrift 140 (1998), 43, S. 12-16

Kasten (2006): Erich Kasten, Body-Modification, München 2006

Kistemann/Exner (2000): Thomas Kistemann, Martin Exner, Bedrohung durch Infektionskrankheiten? Risikoeinschätzung und Kontrollstrategien, Deutsches Ärzteblatt 97 (2000), S. 251-255

Krause/Bremerich/Sztraka (2000): H.-R. Krause, A. Bremerich, M. Sztraka, Komplikationen nach Piercing im Mund und im Gesicht, Mund-, Kiefer-, Gesichtschirurgie 4 (2000), S. 21-24

Laukien (2003): Michael Laukien, Alles über Piercing, Mannheim 2003

Kardinal Lehmann (2005): Kardinal Karl Lehmann, Gegen den Schönheitswahn, in: Bundesministerium für Gesundheit und Soziale Sicherung (Hrsg.), Spieglein, Spieglein an der Wand. Zur Diskussion um den Schönheitswahn, Berlin 2005, S. 12-15

Lotz (1997): Jürgen Lotz, Auf der Suche nach dem Ursprung: Kreativität zwischen Reflex und Reflexion. Punks Das bunte Elend, in: Karl Gröning (Hrsg.), Geschmückte Haut. Eine Kulturgeschichte der Körperkunst, München 1997, S. $234 \mathrm{f}$.

Mall et al. (2002): Julian W. Mall, Christian Pollmann, Joachim M. Müller et al., Keloidbildung des Ohrläppchens nach Ohrlochstechen, Chirurg 73 (2002), S. 514-516

May/Kohnen (2006): Arnd T. May, Tanja Kohnen, Körpermodifikation durch Piercing: Normalität, Subkultur oder Modetrend? Bochum, Medizinethische Materialien Nr. 167, 2006

Mayers et al. (2002): Lester B. Mayers, Daniel A. Judelson, Barry W. Moriarty et al., Prevalence of Body Art (Body Piercing and Tattooing) in University Undergraduates and Incidence of Medical Complications, Mayo Clinic Proceeding 77 (2002), p. 29-34

Meyer (2001): Rüdiger Meyer, Piercing. Gefährlicher Körperschmuck, Deutsches Ärzteblatt 98 (2001), S. 819820

Modest/Fangman (2002): Geoffrey A. Modest, John J. W. Fangman, Nipple Piercing and Hyperprolactinemia, New England Journal of Medicine 347 (2002), p. $1626 \mathrm{f}$.

Muldoon (1997): Kelley A. Muldoon, Body Piercing in Adolescents, Journal Pediatric Health Care 11 (1997), p. 298-301

Peate (2000): Ian Peate, Body piercing: could you answer your patient's queries?, British Journal of Nursing 9 (2000), p. 2163-2168

Roth/Plötz (1998): Reinhold Roth, J. Plötz, Zungenpiercing. Ein Modephänomen nicht ohne anästhesiologische Bedeutung, Anästhesist 47 (1998), S. $690 f$.

Rudolph (2000): Hans Rudolph, Presseinformation Arbeitskreis „Krankenhaus- \& Praxishygiene“ der AWMF, 22.02.2000

Sardella et al. (2002): Andrea Sardella, Massimo Pedrinazzi, Christina Bez et al., Labial piercing resulting gingival recession. A case series, Journal of Clinical Periodontology 29 (2002), p. 961-963 
Scheuer/Lehnen (2002): Bernadette Scheuer, Alexandra Lehnen, Piercing - Ärzte bewegen sich in einer Grauzone, Ärztezeitung, 03.12.2002

Schneider (2004): Anke Schneider, „... damit ich mich spüre ...“. Zur Symptomgenese und Symptomspezifität selbstverletzenden Verhaltens, Berlin 2004

Schildbach (1998): Sebastian Schildbach, Körper- und Intimschmuck aus hausärztlicher Sicht, Journal der Deutschen Gesellschaft für Plastische und Wiederherstellungschirurgie 1998, S. 15-18

Stirn (2002): Aglaja Stirn, Körpermagie, Körpernarzissmus und der Wunsch, Zeichen zu setzen: Eine Psychologie von Tattoo und Piercing, in: Mathias Hirsch (Hrsg.), Der eigene Körper als Symbol? Der Körper in der Psychoanalyse von heute, Gießen 2002

Strametz/Püschel (2001): Sarah E. Strametz, Klaus Püschel, Komplikationen des Piercing, Rechtsmedizin 11 (2001), S. 21-25

Taupitz (2000): Jochen Taupitz, Gutachten A. Empfehlen sich zivilrechtliche Regelungen zur Absicherung der Patientenautonomie am Ende des Lebens?, in: Ständige Deputation des deutschen Juristentages (Hrsg.): Verhandlungen des dreiundsechzigsten Deutschen Juristentages, Band I, 2000, A58-61

The art of Piercing, Ansbach (2005): The art of Piercing, Ansbach, Tatoo \& Piercing in Reutlingen, Piercing 30 (2005), p. 42-44

Türcke (2002): Christoph Türcke, Erregte Gesellschaft: Philosophie der Sensation, München 2002

Vereinigung der Deutschen Plastischen Chirurgie (2005): Vereinigung der Deutschen Plastischen Chirurgie, Pressemeldung vom 14.03.2005

Zbinden (1998): Véronique Zbinden, Piercing. Archaische Riten und modernes Leben, Engerda 1998

Ziegler/Zoschke (1995): Cornelia Ziegler, Barbara Zoschke, Bodypiercing, Wien 1995

Zolondek/Stelling/Hohmann (1998): Ute Zolondek, R. Stelling, H. Hohmann, Entwicklung von Hygieneregeln für das Tätowieren und Piercing und ihre Umsetzung, Gesundheitswesen, 60 (1998), S. 170-172 